\title{
CEST theranostics: label-free MR imaging of anticancer drugs
}

\author{
Yuguo Li ${ }^{1,2, *}$, Hanwei Chen ${ }^{2,3,4, *}$, Jiadi $\mathbf{X u}^{1,2}$, Nirbhay N. Yadav ${ }^{1,2}$, Kannie W. Y. \\ Chan $^{1,2}$, Liangping Luo ${ }^{4}$, Michael T. McMahon ${ }^{1,2}$, Bert Vogelstein ${ }^{5}$, Peter C.M. van \\ Zijl $^{1,2}$, Shibin Zhou ${ }^{5}$ and Guanshu Liu' ${ }^{1,2}$ \\ ${ }^{1}$ F.M. Kirby Research Center for Functional Brain Imaging, Kennedy Krieger Institute, Baltimore, Maryland, USA \\ ${ }^{2}$ The Russell H. Morgan Department of Radiology and Radiological Science, Division of MR Research, Johns Hopkins University \\ School of Medicine, Baltimore, Maryland, USA \\ ${ }^{3}$ Department of Radiology, Panyu Central Hospital, Guangzhou, China \\ ${ }^{4}$ Department of Radiology, The First Affiliated Hospital of Jinan University, Guangzhou, China \\ ${ }^{5}$ Ludwig Center, Howard Hughes Medical Institute and Sidney Kimmel Cancer Center, Johns Hopkins University School of \\ Medicine, Baltimore, Maryland, USA \\ * These authors have contributed equally to this work \\ Correspondence to: Guanshu Liv, email: guanshu@mri.jhu.edu \\ Keywords: CEST, MRI, theranostics, image-guided drug delivery, chemotherapy \\ Received: November 01,2015 Accepted: January 28,2016 Published: February 02, 2016
}

\section{ABSTRACT}

Image-guided drug delivery is of great clinical interest. Here, we explored a direct way, namely CEST theranostics, to detect diamagnetic anticancer drugs simply through their inherent Chemical Exchange Saturation Transfer (CEST) MRI signal, and demonstrated its application in image-guided drug delivery of nanoparticulate chemotherapeutics. We first screened 22 chemotherapeutic agents and characterized the CEST properties of representative agents and natural analogs in three major categories, i.e., pyrimidine analogs, purine analogs, and antifolates, with respect to chemical structures. Utilizing the inherent CEST MRI signal of gemcitabine, a widely used anticancer drug, the tumor uptake of the i.v.-injected, drug-loaded liposomes was successfully detected in CT26 mouse tumors. Such label-free CEST MRI theranostics provides a new imaging means, potentially with an immediate clinical impact, to monitor the drug delivery in cancer.

\section{INTRODUCTION}

Cancer still remains one of the most formidable diseases to cure. Currently, curing unresectable cancers mainly relies on chemotherapies, but the clinical outcome is discouraging, and the patients' quality of life is often poor due to the severe adverse effects. Achieving effective anticancer drug therapy requires not only a certain level of effectiveness of an anticancer drug against specific types of cancer cells, but also the ability to deliver enough of the drug to exceed a threshold effective level of activity over the full anatomic extent of the cancer cell population. The heterogeneity of the tumor often results in unpredictable outcomes in individual patients [1,2]. Thus, it is essential to develop tools with which to assess whether drugs are delivered to the tumor at an adequate concentration and subsequently adjust the treatment plan accordingly, a so-called "personalized medicine" strategy [3], in which non-invasive imaging modalities are expected to play a central role. Currently, there is an extensive investment in the development of molecular imaging techniques that can assess the effectiveness of drug delivery to the tumor [4]. One technical hurdle for the implementation of these approaches, however, is the requirement to chemically or physically attach imaging probes to the drug molecules or drug carriers, which may hamper clinical translation.

In this present study, Chemical Exchange Saturation Transfer (CEST) MRI [5-7] is utilized to directly detect non-chemically labeled chemotherapeutic agents. CEST contrast agents, unlike the commonly used $\mathrm{T} 1$ and $\mathrm{T} 2$ contrast agents, do not rely on the use of paramagnetic labels (i.e., $\mathrm{Gd}, \mathrm{Fe}$, or $\mathrm{Mn}$ ), which makes the use of highly biocompatible, diamagnetic compounds possible. As shown in Figure 1, CEST MRI contrast is generated by the continuous application of RF saturation pulses at the resonance of exchangeable protons in the CEST agent and results in saturated protons (protons with nulled NMR signal), which, due to the nature of the proton 
exchange, are continuously transferred to the surrounding water, resulting in the saturation of a significant portion of the water MR signal. Practically, the requirement for a diamagnetic compound to be CEST MRI-detectable is to have water-exchangeable protons with a slow-tomoderate exchange rate (i.e., $\mathrm{k}_{\mathrm{ex}}<<\Delta \omega$, where $\mathrm{k}_{\mathrm{ex}}$ is the exchange rate and $\Delta \omega$ is the frequency difference between the chemical shifts of a given exchangeable proton and the water protons) and an offset sufficiently far from the water proton resonance to avoid interference of large direct saturation effects, which are often the case for a wide array of medically relevant compounds, including glucose [8,9], glutamate [10] and peptides [11], proteins [12], pyrimidine compounds [13] and even therapeutic bacterial cells [14]. Based on our previous studies on the relationship between CEST properties and chemical structures, we hypothesized that CEST MRI could be used for the label-free detection of many anticancer drugs using their inherent exchangeable protons, in hydroxyl $(\mathrm{OH})$, amide $(\mathrm{NH})$, and amine $\left(\mathrm{NH}_{2}\right)$ groups

\section{RESULTS AND DISCUSSION}

\section{Cytidine analogue anticancer drugs can be directly detected by CEST MRI}

To test our hypothesis, we first examined the four widely used cytidine analogue anticancer drugs, gemcitabine ( $\mathrm{dFdC})$, cytarabine (araC), decitabine (Dec), and azacitidine (Aza), which are either approved or in clinical trials, and their natural analog deoxycytidine (dC). As shown in Figure 2a, all drugs have a chemical structure similar to that of deoxycytidine. Our in vitro results showed that, as expected, all of these anticancer drugs (in PBS solution, $\mathrm{pH}=7.4$ and $37^{\circ} \mathrm{C}$ ) exhibited two strong CEST MRI signals, around 2.0-2.4 ppm and 1.0 ppm, corresponding to the amino and hydroxyl protons respectively. In Figures 2b-2d, CEST signals are shown in both Z-spectra (solid lines), in which the water proton signal is plotted as a function of saturation frequency, and in $\mathrm{MTR}_{\text {asym }}$ plots (dotted lines), a more quantitative metric defined by: MTR ${ }_{\text {asym }}=\left(\mathrm{S}^{-\Delta \omega}-\mathrm{S}^{\Delta \omega}\right) / \mathrm{S}_{0}$, where $\mathrm{S}^{-\Delta \omega}$ and $\mathrm{S}^{\Delta \omega}$ are the MRI signal intensities after saturation at negative and positive values of the offset frequency $\Delta \omega$ from the water proton frequency (set at $0 \mathrm{ppm}$ by convention); $\mathrm{S}_{0}$ is the intensity in the absence of a saturation pulse. Maximal MTR $_{\text {asym }}$ values of $0.119 \pm 0.007$ and $0.129 \pm 0.025$ could be achieved for $20 \mathrm{mM} \mathrm{dFdC}$ at offsets of 2.2-2.3 ppm and $+1.0 \mathrm{ppm}$, respectively, when a continuous wave $\mathrm{RF}\left(\mathrm{B}_{1}=\right.$ $3.6 \mu \mathrm{T}$ and $\mathrm{t}_{\mathrm{sat}}=4 \mathrm{sec}$ ) saturation pulse was used.

\section{The pH dependence of CEST MRI signal of cytidine-based anticancer drugs}

We measured the CEST contrast of gemcitabine in a $\mathrm{pH}$ range from 2 to 9, as shown in Figure 3. We also used the frequency-labeled exchange (FLEX) transfer method
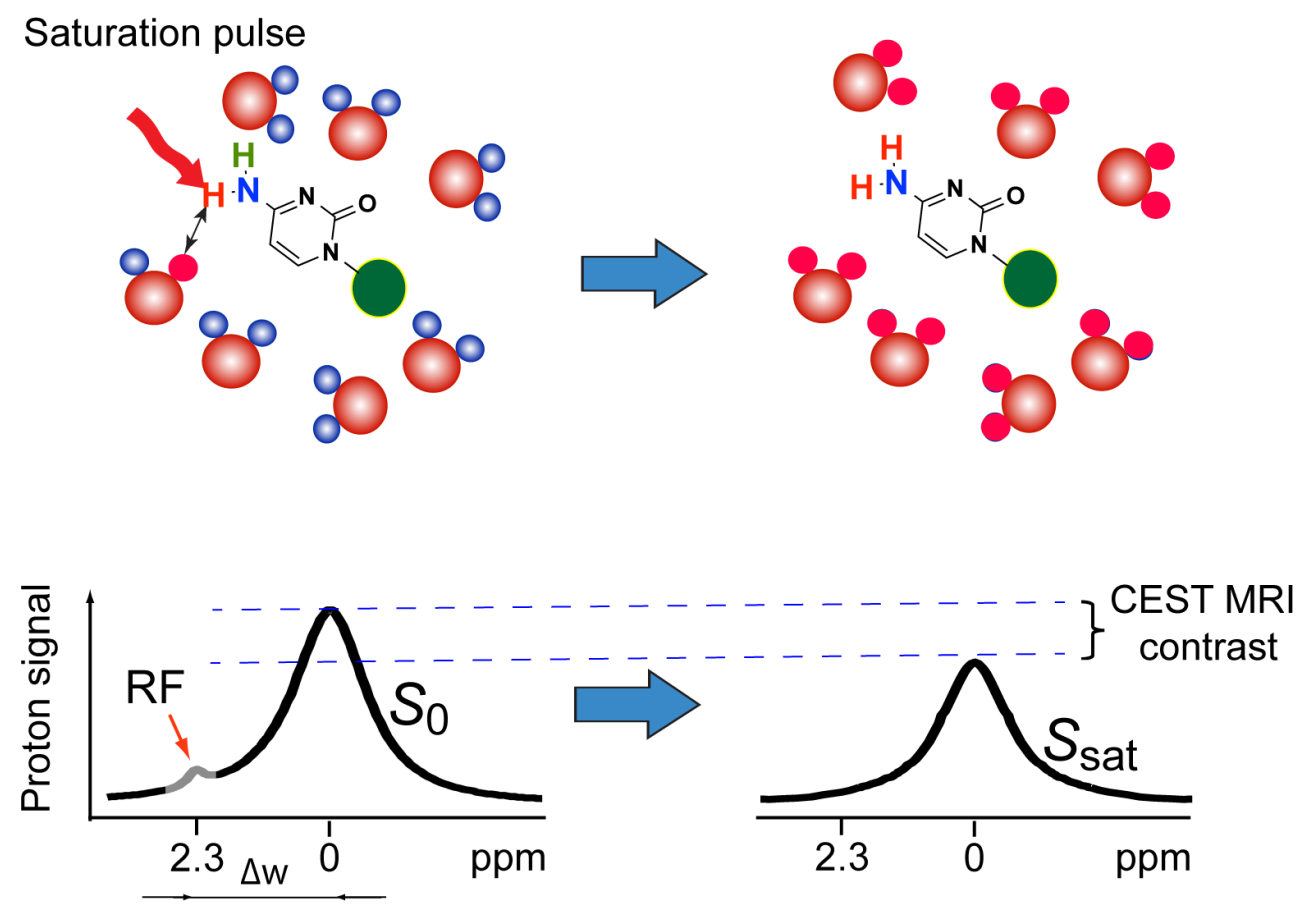

Figure 1: The principle of CEST MRI detection of anticancer drugs, such as gemcitabine. Exchangeable protons on the drug molecules can transfer RF saturation to the protons of surrounding water (top row), resulting in a decrease in MRI signal. Continuously applying RF pulses leads to the saturation of more water protons, generating a detectable MRI contrast called Chemical Exchange Saturation Transfer (CEST) contrast (bottom row). 
as previously described $[13,15]$ to determine the exchange rate of the amine protons of $\mathrm{dFdC}$ at different $\mathrm{pH}$. The results revealed a strong $\mathrm{pH}$ effect on the CEST contrast of hydroxyl protons. For example, the CEST MRI signal of $\mathrm{OH}$ protons increases dramatically ( $>2$ times) when the $\mathrm{pH}$ drops from $\mathrm{pH} 8$ to $\mathrm{pH} 6$. In contrast, the CEST contrast of $\mathrm{NH}_{2}$ is relatively stable in $\mathrm{pH}$ range from 6.5 to 7.5. For this reason, we chose the CEST MRI signal of $\mathrm{NH}_{2}$ (i.e., $\sim 2.3 \mathrm{ppm}$ in the $\mathrm{pH}$ range of 6.5 to 7.5 ) for quantifying dFdC. Similar $\mathrm{pH}$ dependences for other drugs were observed (Figure S2).

Interestingly, at $\mathrm{pH} 3$, the $\mathrm{CEST}$ contrast of $\mathrm{OH}$ is completely gone while that of $\mathrm{NH}_{2}$ is shifted from $2.2 \mathrm{ppm}$ to $3.4 \mathrm{ppm}$ (Figure $3 \mathrm{~b}$ ). The $\mathrm{pKa}$ of the amine protons of $\mathrm{dFdC}$ was estimated to be 4.3 (Table S1, calculated using Advanced Chemistry Development (ACD/Labs) Software V11.02). Hence, the shift of amine CEST is likely due to the protonation of $\mathrm{NH}_{2}(\mathrm{pKa}=4.3)$ and the protonated $\mathrm{NH}^{3+}$ having a new chemical shift at $\sim 3.4 \mathrm{ppm}$. However, it may stem from the $\mathrm{OH}$ protons of sugar [9]. The exact assignment of this chemical shift is still under investigation.

\section{Sensitivity of the CEST MRI detection}

To evaluate the minimal concentration for using CEST MRI to detect gemcitabine, we performed CEST MRI on the samples containing gemcitabine at a concentration ranging from $0.5 \mathrm{mM}$ to $20 \mathrm{mM}$. To mimic the in vivo baseline magnetization transfer effect, we also prepared samples in $1 \%$ or $2 \%$ agarose gel. The results are shown in Figure 4. Figure 4a shows that the MTR $_{\text {asym }}$ values at $2.3 \mathrm{ppm}$ and $1.0 \mathrm{ppm}$, for $\mathrm{NH}_{2}$ and $\mathrm{OH}$ protons respectively, have a very good linearity in the concentration below $10 \mathrm{mM}$, indicating that the $\mathrm{MTR}_{\text {asym }}$ can be used directly to quantify concentration. Figure $4 \mathrm{~b}$ shows the CEST signal of $\mathrm{NH}_{2}$ protons of $\mathrm{dFdC}$ at low concentrations (i.e., 0.5 to $2 \mathrm{mM}$ ) in $1 \%$ and $2 \%$ agarose gel phantoms. Interestingly, agarose gel itself has a small but detectable CEST signal, suggesting it may be a better phantom to determine the minimum detection sensitivity by mimicking the in vivo condition.

We used two methods to determine the minimum detection sensitivity. First, we performed a statistical

\section{a Natural Nucleoside}
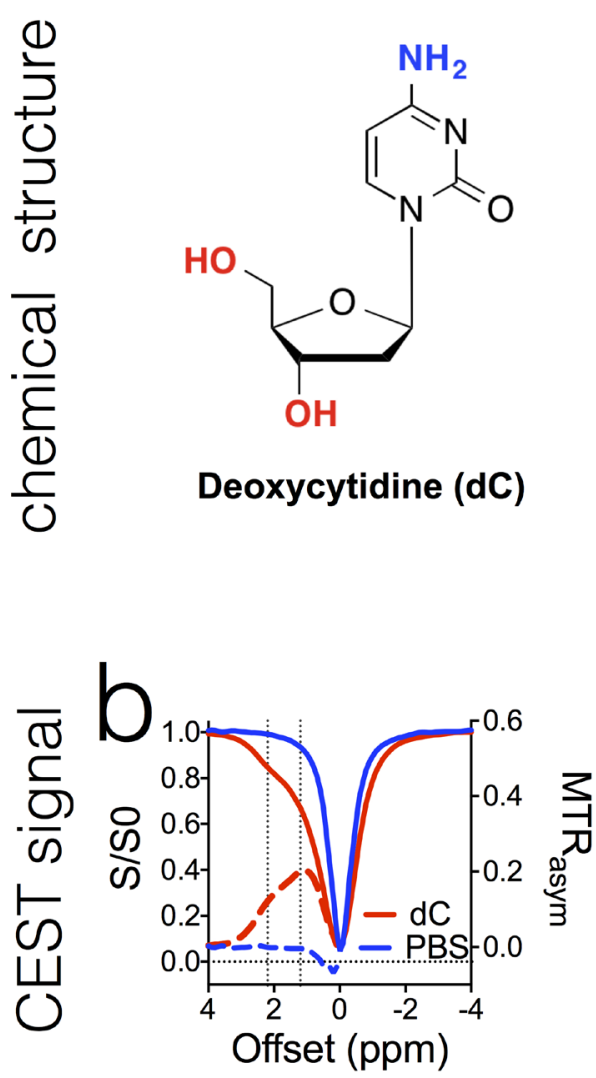

\section{Chemotherapeutic agents}

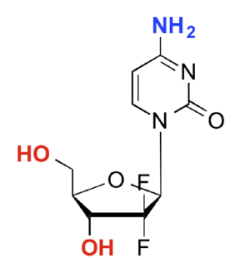

Gemcitabine (dFdC)

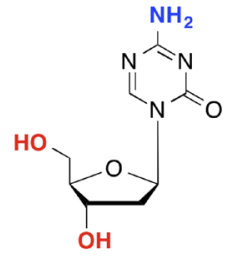

Decitabine (Dec)

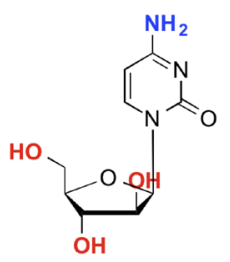

\section{Cytarabine (araC)}

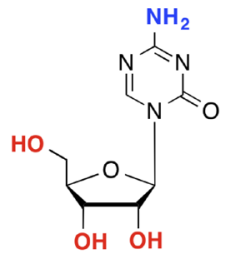

Azacitidine (Aza)
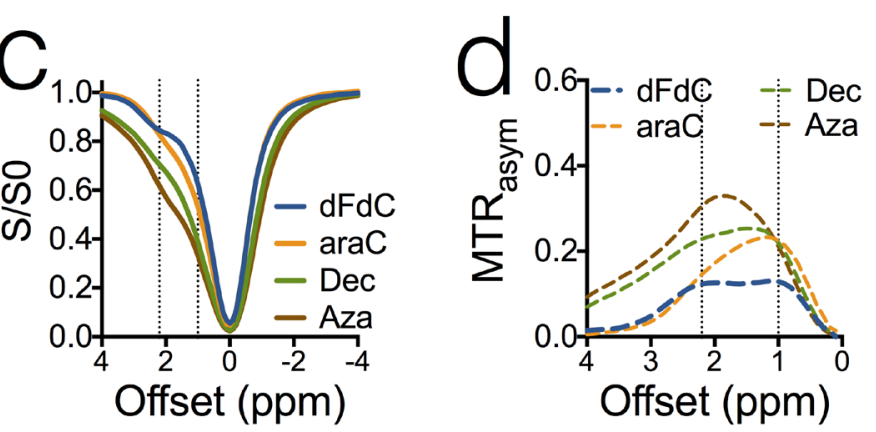

Figure 2: The chemical structure of cytidine- based agents (a) and their CEST MRI contrast, as shown both by z-spectra (b \& c) and MTR $_{\text {asym }}$ plots (b \& d). All samples were prepared in PBS (pH 7.4) at a concentration of $20 \mathrm{mM}$ and measured at $37^{\circ} \mathrm{C}$ using a $3.6 \mu \mathrm{T}, 3 \mathrm{sec} \mathrm{CW}$ RF pulse. 

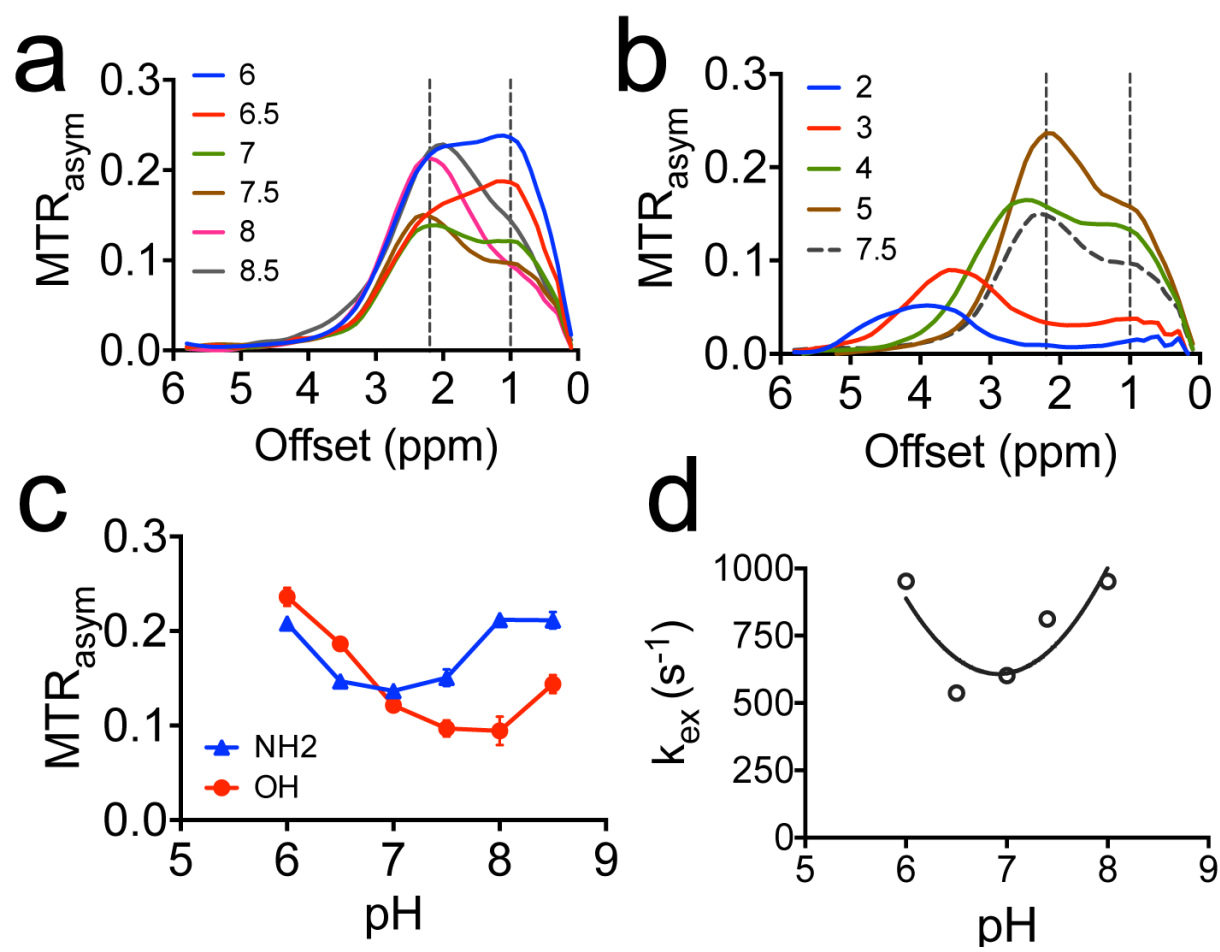

Figure 3: The pH dependence of CEST contrast of dFdC. a. The $\mathrm{MTR}_{\text {asym }}$ plots of $20 \mathrm{mM} \mathrm{dFdC} \mathrm{in} \mathrm{the} \mathrm{pH}$ range from 6 to 8.5 ; b. The $\mathrm{MTR}_{\text {asym }}$ plots of $20 \mathrm{mM} \mathrm{dFdC} \mathrm{in} \mathrm{a} \mathrm{pH}$ range from 2 to 5 . The $\mathrm{pH} 7.5$ is also plotted as a reference; $\mathbf{c}$. The pH dependence of CEST contrast of amine and hydroxyl in a $\mathrm{pH}$ range from 6.0 to 8.5 ; d. The $\mathrm{pH}$ dependence of the exchange rate of $\mathrm{NH} 2$ in a $\mathrm{pH}$ range from 6.0 to 8.0 using the FLEX method.
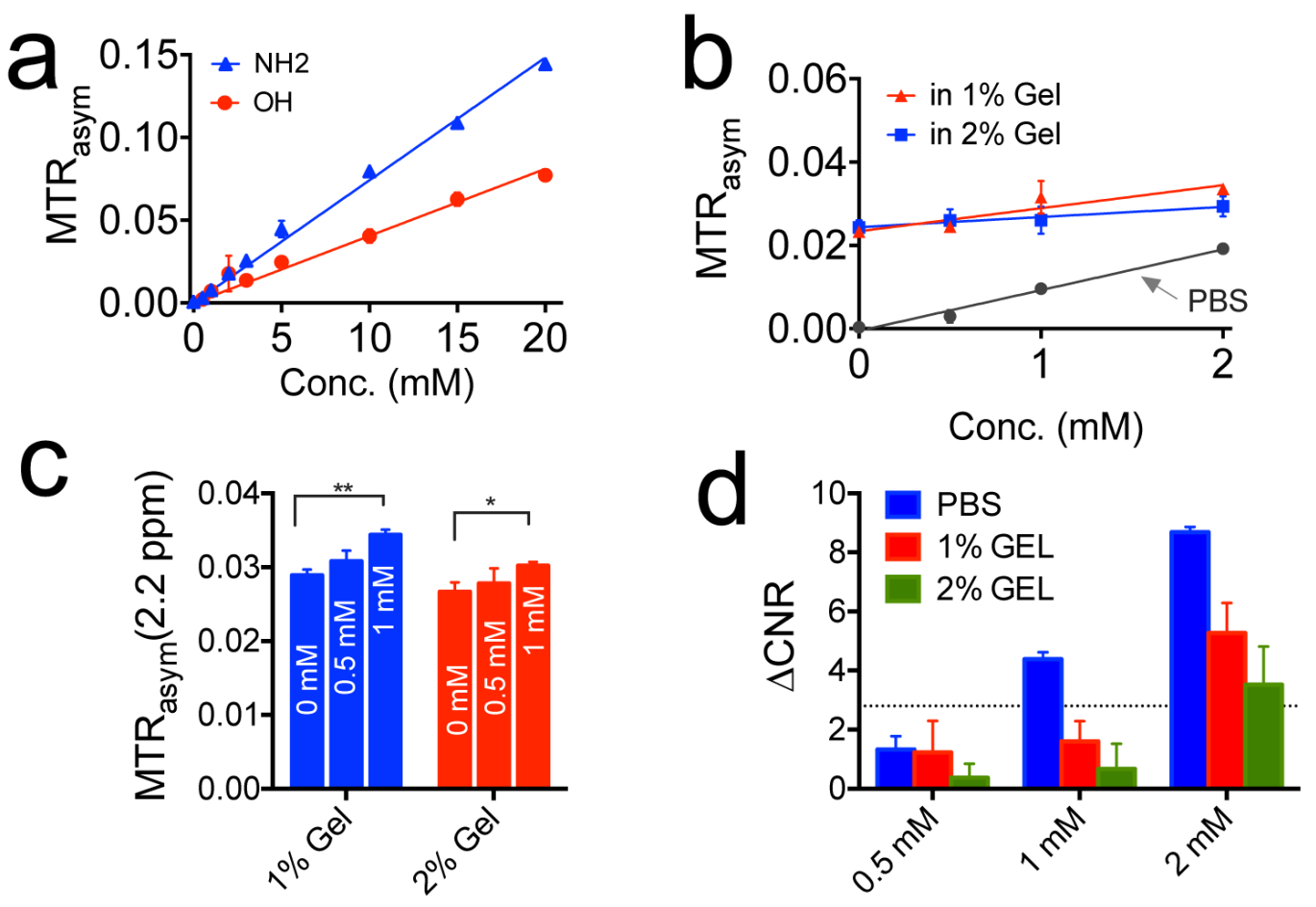

Figure 4: The detection limit of CEST MRI for detecting gemcitabine. a. The calibration curves of the CEST signal of gemcitabine at concentrations ranging from 0.5 to $20 \mathrm{mM}$ in PBS; b. The CEST MRI contrast at 2.2 ppm for samples prepared in $1 \%$ and $2 \%$ agarose gels, the CEST contrast in PBS is shown for comparison; c. Bar plots of the CEST contrast at different concentrations in each sample. A two-tailed paired Student's t-test was performed $(* * p<0.01$ and $* P<0.05)$. The results are mean $\pm \operatorname{SD}(n=3)$; d. Bar plots of the $\triangle \mathrm{CNR}$ for samples containing gemcitabine at different concentrations as compared to reference samples containing $1 \%$ or $2 \%$ agarose gel. The horizontal dashed line represents $\Delta \mathrm{CNR}=2 \sqrt{2}$. 
analysis (the two-tailed, unpaired Student's $t$ test, $n=3$ ) to determine the minimum concentration of gemcitabine that can generate a significant difference in CEST signal between just agarose gel and gemcitabine containing agarose gel. As shown in Figure 4c, a significant difference could be achieved for samples containing 1 $\mathrm{mM}$ or higher gemcitabine but not those containing 0.5 $\mathrm{mM}$ gemcitabine. The $\mathrm{P}$ values were determined to be 0.0007 and 0.0106 for $1 \mathrm{mM}$ gemcitabine in $1 \%$ and $2 \%$ agarose gel respectively, with both showing significant differences (i.e., $P<0.05$ ) compared to the reference gel samples. Second, we also adapted a previously published contrast-to-noise Ratio (CNR)-based approach [16] to determine the detection threshold in the presence of systemic noise. As shown in Figure 4d, when a $\triangle \mathrm{CNR}$ threshold of $2 \sqrt{2}$ was used [16], the minimum detection sensitivity was estimated to be $<1 \mathrm{mM}$ for PBS samples

\section{Chemical structure}<smiles>Nc1nc(F)nc2c1ncn2COC(O)C(O)COP(=O)([O-])[O-]</smiles>

and 1-2 mM for gel samples. It should be noted that the estimation of CEST detectability is affected by the choice of CNR threshold and CNR thresholds in different range (i.e., 0.6-2) have been reported previously [17, 18]. When a $\triangle \mathrm{CNR}$ threshold $=1$ was used, for instance, the detection limit was estimated to be $0.5 \mathrm{mM}$ for gemcitabine in $1 \%$ agarose gel. In comparison, the typical detectability for small molecular Gd-based contrast agents is in the range of hundreds $\mu \mathrm{M}$ to $\mathrm{mM}$ [19] and that of ${ }^{19} \mathrm{~F}$ MRI is in the range of tens of $\mathrm{mM}$ [20]. Our study suggests that CEST MRI can provide a similar detectability as those imaging contrast generation strategies.

It also should be noted that the results shown in Figure 4 were acquired using a $3.6 \mu \mathrm{T}, 3$-second $\mathrm{CW}$ saturation pulse at a spatial resolution of 130x130 $\mu \mathrm{m}^{2}$. Because the saturation parameters significantly affect the $\mathrm{MTR}_{\text {asym }}$, a new, saturation-parameter-independent

\section{CEST MRI contrast}

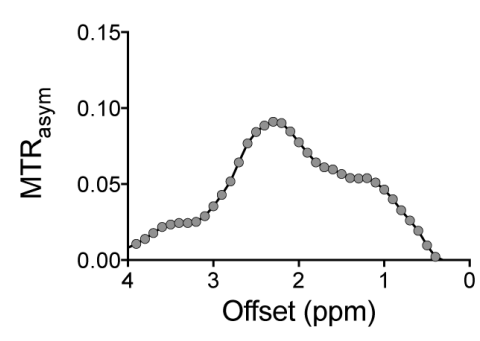

\section{Purine analogue (fludarabine)}<smiles>CN(Cc1cnc2nc(N)nc(N)c2n1)c1ccc(C(=O)N[C@@H](CCC(=O)[O-])C(=O)[O-])cc1</smiles>

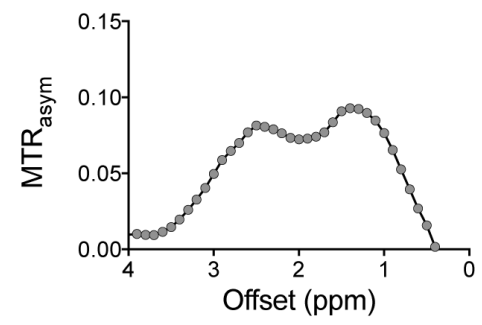

\section{Antifolate (methotrexate)}<smiles></smiles>

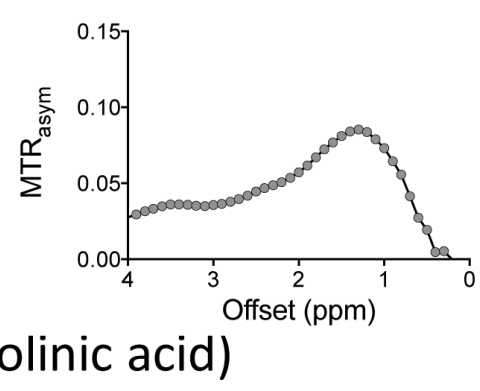

Figure 5: Example of other CEST MRI detectable chemotherapeutic agents. All samples were prepared in PBS (pH 7.4) at a concentration of $20 \mathrm{mM}$ and measured at $37^{\circ} \mathrm{C}$ using a $3.6 \mu \mathrm{T}, 3 \mathrm{sec} \mathrm{CW}$ RF pulse. 
metric for quantifying CEST contrast may be more useful to determine the detectability (or sensitivity) of a CEST agent.

\section{CEST MRI can be used to detect antitumor drugs in other categories}

We then expanded our approach to other antitumor drug categories. Among the twenty-two anticancer drugs (Table S4) that we investigated, antifolates (e.g., methotrexate and the drug modulator folinic acid) and purine analogs (e.g., fludarabine) showed good CEST MRI detectability (Figure 5). These results imply that CEST MRI can be used to detect any drug that has exchangeable protons (amides, amines and hydroxyls) at the appropriate exchange rate, hence has a widespread application.

Compounds sharing similar chemical structures showed similar CEST signals, indicating the possibility to predict the CEST properties based on the chemical structure of a given drug, as suggested in previous studies [13]. Thus, the properties of exchangeable protons can be substantially affected by surrounding chemical modification, which results in changes in the CEST signal. For example, when pyrimidine is replaced by triazine, the $\mathrm{NH}_{2}$ protons of decitabine (Dec) and azacitidine (Aza) show much stronger CEST effects (i.e., MTR ${ }_{\text {asym }}(2.3$ $\mathrm{ppm})=0.23$ and 0.31 , respectively) than that of $\mathrm{dC}$ (i.e., $\left.\operatorname{MTR}_{\text {asym }}(2.1 \mathrm{ppm})=0.12\right)$. In contrast, the CEST signal of hydroxyl protons appears to be mainly affected by the number of protons.

\section{Use CEST MRI to monitor liposome-mediated drug delivery to the tumor}

The direct visualization of drugs using CEST MRI should allow the label-free tracking of a nanoparticle drug delivery system. To demonstrate this, we encapsulated gemcitabine into liposomes (i.e., liposomal $\mathrm{dFdC}$ ) and used CEST to monitor the tumor uptake of liposomes in an experimental tumor model. The liposomal $\mathrm{dFdC}$ was prepared using a procedure described previously [21, 22] and a liposomal formulation (DPPC: cholesterol: DPPE$\mathrm{PEG}=55: 45: 5)$ [23], with an additional $0.5 \%$ rhodamineB-PE (fluorescent dye). The starting solution contained $50 \mathrm{mg} / \mathrm{ml}$ gemcitabine hydrochloride $(\mathrm{pH} \sim 3)$. The size of formed liposomes was measured to be $\sim 120 \mathrm{~nm}$ using a dynamic light scattering Nanosizer. The encapsulation rate was estimated as $\sim 30.8 \%$, using the UV absorbance at $268.8 \mathrm{~nm}$ of $\mathrm{dFdC}$. Our in vitro drug release assay showed that the initial release of $\mathrm{dFdC}$ was very rapid, i.e., $\sim 34 \%$ of loaded $\mathrm{dFdC}$ within the first three hours of dialysis. The concentration of intra-liposomal $\mathrm{dFdC}$ was then stable and only decreased $4.4 \%$ over a period of 24 hours (Figures S4). As shown in Figure 6, the encapsulation of dFdC in liposomes doesn't have a noticeable impact on the CEST properties, as evidenced by the similarity between the shape of the MTR asym plot of liposomal $\mathrm{dFdC}$ and that of free form, at both $\mathrm{pH} 7.4$ and $\mathrm{pH}$ 3.0.

We then performed CEST MRI on CT26 tumorbearing mice before and after the injection of liposomal $\mathrm{dFdC}$. To enhance the intratumoral accumulation of systemically administered liposomes, mice also received a co-treatment of TNF- $\alpha$, which was shown previously to increase the tumor permeability substantially [24, 25]. Figure 7 shows that liposomal $\mathrm{dFdC}$ could be readily detected in TNF- $\alpha$-treated CT26 tumors five hours after a tail vein injection of $20 \mathrm{mg}$ lipid/kg b.w. (c.a. $80 \mathrm{mg}$ $\mathrm{dFdC} / \mathrm{kg}$ b.w.) and TNF- $\alpha$ (1 $\mu \mathrm{g}$ per mouse). A relatively uniform elevation of CEST MRI signal at $3.2 \mathrm{ppm}$ was conspicuous at five hours after the injection (Figure 7a). The accumulation of liposomal $\mathrm{dFdC}$ in the tumor resulted in a net increase of 0.015 in $\mathrm{MTR}_{\text {asym }}$ (Figures $7 \mathrm{~b}$ and $7 \mathrm{c}$ ) as compared to that before the injection. The average increase of CEST contrast in the three tumors studied (Figure 7d) was $0.022 \pm 0.012$ (the paired two-tailed Student's t test: $P<0.05, n=3)$. The CEST MRI detection of tumor uptake of liposomal $\mathrm{dFdC}$ was validated using fluorescence imaging (Figures 7f, S7\&S8). Our results suggest that our approach is capable of directly monitoring delivery of nanoparticulate chemotherapeutics. Moreover,

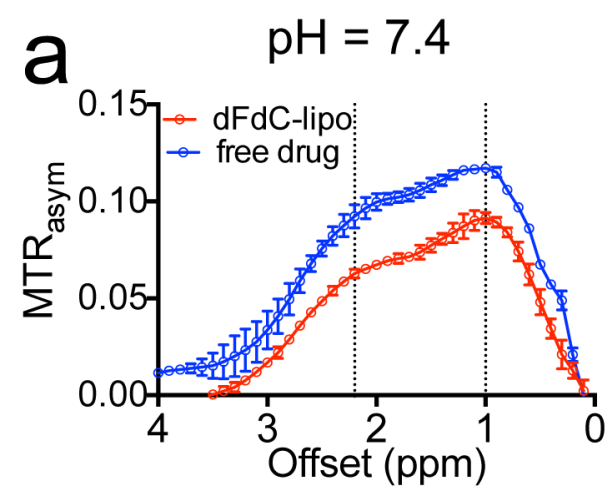

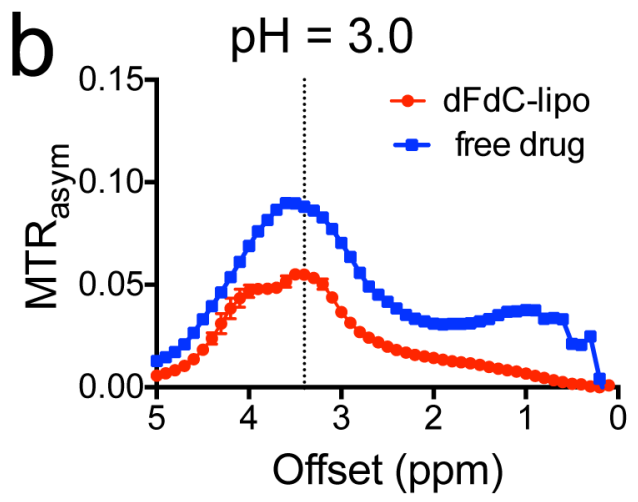

Figure 6: The CEST of liposomal dFdC ( $\sim 80 \mathrm{nM}$ per liposome) and compared with that of free drug (10 $\mathrm{mM})$ at a. $\mathrm{pH}$ 7.4 and b. $\mathrm{pH} 3.0$. 
as TNF- $\alpha$ is being investigated clinically for improving the drug delivery of chemotherapy [26], this approach may also be useful for assessing the tumor responses to the combination of nanomedicine and TNF- $\alpha$ or other vascular-targeting treatment.

The capability to directly track drug-loaded nanoparticles without additional imaging labels is expected to be very helpful for both pre-clinical development and the clinical use of nanoparticulate chemotherapeutic agents. There are more than 45 nanoparticulate drug formulations that have been clinically approved, and at least 200 products are currently in Phase I-III clinical trials [27]. If some of them can be tailored into theranostic (therapeutic and diagnostic [28]) systems via the proposed CEST MRI method, they can be used directly in the clinic to stratify patients and enable personalized medicine.
Moreover, the high local concentration of the drugs loaded in the nanoparticle carriers also helps to boost the sensitivity of CEST, potentially can be used to lessen the requirement of low $\mathrm{mM}$ concentration drugs for the CEST MRI detection. Thus our application should have a great impact to nanomedicine in addition to traditional chemotherapies. One limitation of our method is that it is currently unable to detect small molecular drugs at therapeutic concentrations (e.g. $\sim \mu \mathrm{M}$ ). We will continue to work on improving the detectability of the proposed approach with the help of novel CEST techniques [19, 29, $30]$ in an effort to broaden application. Another technical challenge is the translation of our methods developed on $9.4 \mathrm{~T}$ and $11.7 \mathrm{~T}$ small animal scanners to clinical $3 \mathrm{~T}$ scanners. While currently many groups are working on this issue and there are indeed a handful of recently developed
2
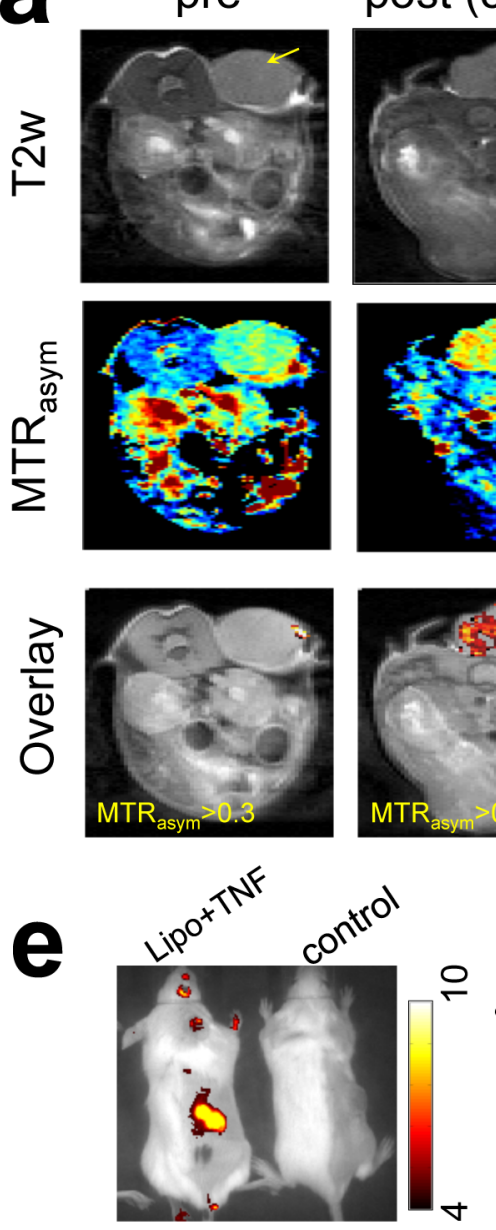
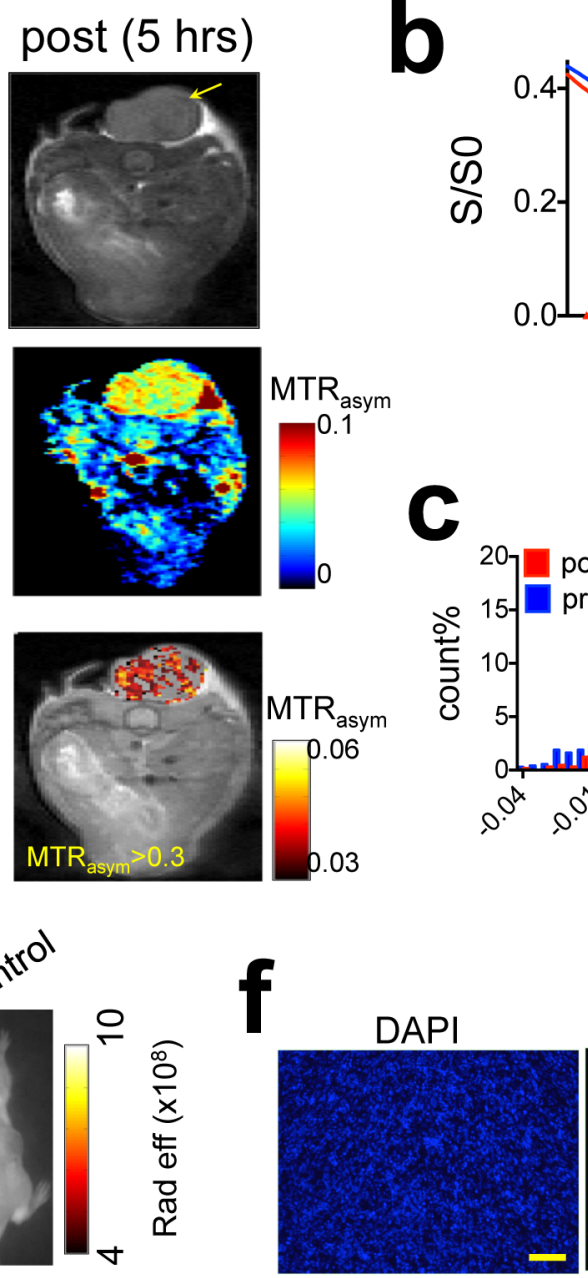
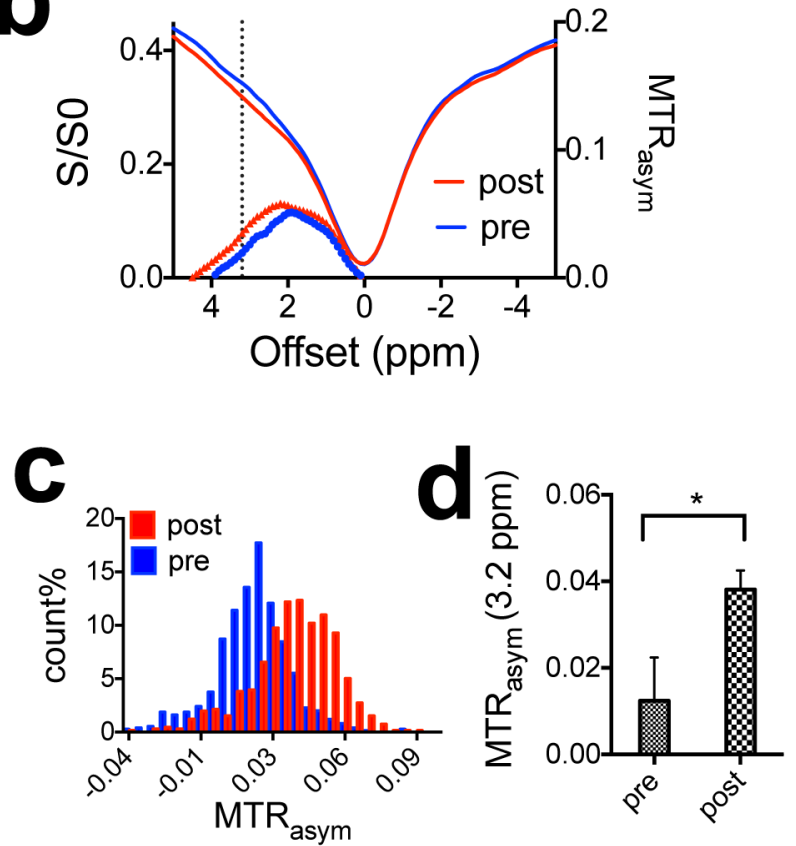

Figure 7: In vivo detection of the tumor uptake of liposomal gemcitabine using CEST theranostics. a. CEST MRI detection of a CT26 tumor (co-treated with TNF- $\alpha$ ) before and five hours after the i.v. injection of liposomal dFdC. From top to bottom: T2w images; CEST maps at $~ 3.2 \mathrm{ppm}$; and CEST/T2w overlaid images (only the CEST in the tumor region is shown). b. Mean tumor CEST signal $\left(\mathrm{MTR}_{\text {asym }}\right.$ plots an Z spectra) before and after the injection. c. Histogram of the MTR ${ }_{\text {asym }}$ values within the tumor regions, before and after the injection of liposomes. d. Statistical analysis of mean CEST signal changes in the tumor regions after the injection $(n=3)$. e. Whole body fluorescence imaging of a representative mice injected with liposomal dFdC (Gem-lipo) in the presence TNF- $\alpha$, and a control mouse (saline injection). f. Histopathology of tumor section, clearly showing the accumulation and distribution of rhodamine-B-labeled liposomes in the tumor co-injected with liposomes and TNF- $\alpha$. Nuclei are stained with DAPI (blue). (Scale bar $=100 \mu \mathrm{m})$. 
CEST MRI methods, including APT [12], gluCEST [10], glucoCEST [8,9], and acidoCEST [31], that have been successfully translated, this will need to be demonstrated for the approach suggested here.

\section{MATERIALS AND METHODS}

\section{MRI}

In vitro CEST images were acquired on a 9.4T Bruker Avance system equipped with a $15 \mathrm{~mm}$ sawtooth RF coil. A modified RARE sequence $(T R=6.0 \mathrm{sec}$, effective TE $=43.2 \mathrm{~ms}$, RARE factor $=16$, slice thickness $=0.7 \mathrm{~mm}, \mathrm{FOV}=14 \times 14 \mathrm{~mm}$, matrix size $=128 \times 64$, resolution $=0.11 \times 0.22 \mathrm{~mm}^{2}$, and $\left.\mathrm{NA}=2\right)$ [16] including a magnetization transfer (MT) module (one CW pulse, $\mathrm{B} 1=3.6 \mu \mathrm{T}(150 \mathrm{~Hz}), 3 \mathrm{sec})$ was used to acquire CEST weighted images from $-10 \mathrm{ppm}$ to $10 \mathrm{ppm}(\mathrm{step}=0.2 \mathrm{ppm})$ around the water resonance $(0 \mathrm{ppm})$ [32]. The absolute water resonant frequency shift was measured using the WAter Saturation Shift Reference (WASSR) [33] method modified with Lorentzian analysis. The same parameters as in CEST imaging were used except TR $=1.5 \mathrm{sec}$, $\mathrm{t}_{\text {sat }}=500 \mathrm{~ms}, \mathrm{~B}_{1}=0.5 \mu \mathrm{T}(21.3 \mathrm{~Hz})$ and the saturation frequency swept from $-1 \mathrm{ppm}$ to $1 \mathrm{ppm}(\mathrm{step}=0.1 \mathrm{ppm})$.

In vivo images were acquired on an $11.7 \mathrm{~T}$ Bruker Biospec horizontal bore scanner (Bruker Biosciences, Billerica, MA) equipped with a $23 \mathrm{~mm}$ Circular Polarized MRI transceiver volume coil. The same imaging scheme described above was used with the addition of a fat suppression pulse $(3.4 \mathrm{~ms}$ hermite pulse, offset $=-3.5$ $\mathrm{ppm})$. The acquisition parameters were: $\mathrm{TR}=5.0 \mathrm{sec}$, effective TE $=6 \mathrm{~ms}$, RARE factor $=10, \mathrm{t}_{\mathrm{sat}}=3 \mathrm{sec}, \mathrm{B}_{1}$ $=3.6 \mu \mathrm{T}(150 \mathrm{~Hz})$, slice thickness $=1 \mathrm{~mm}$, acquisition matrix size $=128 \times 64, \mathrm{FOV}=20 \times 20 \mathrm{~mm}$, and $\mathrm{NA}=2$. Due to the $\mathrm{B}_{0}$ field inhomogeneity, we incremented the saturation offset $\pm 1 \mathrm{ppm}$ (0.1 ppm steps) with respect to water for $\mathrm{B}_{0}$ mapping.

Data processing was performed using customwritten scripts in MATLAB (Mathworks, Waltham, MA). CEST spectra were calculated from the mean of an ROI placed over each sample after $\mathrm{B}_{0}$ correcting the contrast on a per voxel basis. The CEST signal was quantified using $\mathrm{MTR}_{\text {asym }}$ at particular offsets of interest (i.e. $\Delta \omega=$ $+2.2 \mathrm{ppm})$ using the definition: $\mathrm{MTR}_{\text {asym }}=\left(\mathrm{S}^{-\Delta \omega}-\mathrm{S}^{+\Delta \omega}\right) /$ $\mathrm{S}_{0}$, where and $\mathrm{S}^{[-\Delta \omega,+\Delta \omega]}$ is the water signal intensity in the presence of saturation pulse at offsets $\pm \Delta \omega$, and $\mathrm{S}_{0}$ is the water signal intensity in the absence of saturation pulses.

\section{Preparation and characterization of liposomal drugs}

DPPC (Avanti Polar Lipids), cholesterol, and DPPE-PEG 2000 (Avanti Polar Lipids) (molar ratios
50:45:5) [23] with an additional $0.5 \%$ rhodamine-BPE (fluorescent dye) were dissolved in chloroform (2 $\mathrm{mL}$ ). The solvent was removed in vacuum to give a thin lipid film, which was hydrated by shaking in $50 \mathrm{mg} /$ $\mathrm{ml}$ gemcitabine hydrochloride $(\mathrm{pH} \sim 3)$ at $50{ }^{\circ} \mathrm{C}$ for 2 $\mathrm{h}$. The vesicle suspension was sonicated for 30 seconds and then extruded successively through 0.4 and $0.1 \mu \mathrm{m}$ polycarbonate membranes to obtain the final liposomes with low polydispersity at the desired size. The average size and polydispersity index were then measured by dynamic light scattering experiments on a Zetasizer Nano ZS90 (Malvern Instruments, Southborough, MA). The liposomes were then filtered through Sephadex G-50 gel columns (GE Healthcare Life Sciences, Pittsburg, PA) twice to remove unloaded drugs, and stored at $4{ }^{\circ} \mathrm{C}$ prior to use. The average size of liposomes was measured as $\sim 120 \mathrm{~nm}$ and final lipid concentration was about $20 \mathrm{mg}$ lipid /ml.

\section{Animals}

All experiments conducted with mice were performed in accordance with protocols approved by the Johns Hopkins University Institutional Animal Care and Use Committee (IACUC). CT26 (CRL-2638) murine colorectal adenocarcinoma cells were purchased from the American Type Culture Collection (ATCC) and grown in McCoy's 5A Medium (Invitrogen/Life Technologies, Carlsbad, CA) supplemented with $10 \%$ Fetal Bovine Serum (FBS, HyClone, Thermo Scientific, Waltham, MA) at $37{ }^{\circ} \mathrm{C}$ with $5 \% \mathrm{CO}_{2}$. Five million CT26 cells were injected subcutaneously into the right flank of female BALB/c mice (6-8 weeks; Harlan, Indianapolis, $\mathrm{IN} ; \sim 20 \mathrm{~g}$ in weight), and allowed to grow for $\sim 10$ days. Ten days after implantation, mice $(\mathrm{n}=3$, each group) received a tail vein injection of $100 \mathrm{mg}$ lipid/ $\mathrm{kg}$ b.w. (c.a. $80 \mathrm{mg}$ gemcitabine $/ \mathrm{kg}$ b.w.) with or without TNF- $\alpha$ (1 $\mu \mathrm{g}$ per mouse). TNF- $\alpha$ was reconstituted freshly before administration in doubly- distilled $\mathrm{H}_{2} \mathrm{O}$ at $100 \mu \mathrm{g} /$ $\mathrm{mL}$ and diluted into $0.1 \%(\mathrm{w} / \mathrm{v}) \mathrm{BSA}$ in PBS at a final concentration of $10 \mu \mathrm{g} / \mathrm{mL}$. Liposomal gemcitabine was injected within a few minutes thereafter. The CEST images were acquired at 4-5 hours after the injection.

\section{Fluorescence imaging}

Fluorescence imaging was performed and analyzed using a Spectrum/ CT IVIS ${ }^{\circledR}$ in vivo imaging system with the Living Image ${ }^{\circledR}$ software (PerkinElmer, Waltham, MA). Fluorescence signal $($ emission $=620 \mathrm{~nm}$, excitation $=570$ $\mathrm{nm}$ ) was quantified as radiant efficiency. 


\section{Immunohistochemistry}

Excised tumors were imaged immediately after MRI measurements and processed for histology. Tumor sections of $10 \mu \mathrm{m}$ were stained with 4',6-diamidino-2phenylindole (DAPI) for nuclei and examined under an inverted microscope (Olympus, Tokyo, Japan) for DPAI (blue) and rhodamine-B conjugated with liposomes (red).

\section{Statistics}

All in vitro experiments reported were performed in triplicate. Quantitative data are expressed as mean $\pm \mathrm{SD}$, as indicated. Statistical significance was assessed by the two-tailed unpaired Student's $t$-test. Values of $P<0.05$ were considered significant and asterisked.

\section{CONCLUSIONS}

In summary, we have demonstrated a labelfree imaging approach to "see" drugs directly, namely CEST theranostics. We screened a wide array of chemotherapeutic agents in vitro and confirmed the CEST MRI contrast of the drugs and their nontoxic analogs in three major categories: pyrimidine analogs, purine analogs, and antifolates. We also showed that CEST MRI could be used synergistically with nanomedicine to transform currently available therapeutics directly into theranostics, which enabled the first successful CEST MRI detection of the tumor uptake of liposomal gemcitabine without need for synthetic imaging labels. These results imply that we can potentially transform many currently available drugs, including those already in the clinic and those still under pre-clinical development, to be MRIdetectable theranostic agents, WITHOUT any radioactive-, paramagnetic-, or super-paramagnetic-based labeling.

\section{GRANT SUPPORT}

This research was supported by NIH grants R21EB008769, R21EB015609, R01EB015031, R01EB015032, P41EB015909 (NIBIB), and S10RR028955 (NCRR).

\section{CONFLICTS OF INTEREST} interests.

All the authors declare no competing financial

\section{REFERENCES}

1. Tredan O, Galmarini CM, Patel K and Tannock IF. Drug resistance and the solid tumor microenvironment. J Natl Cancer Inst. 2007; 99:1441-1454.
2. Olive KP, Jacobetz MA, Davidson CJ, Gopinathan A, McIntyre D, Honess D, Madhu B, Goldgraben MA, Caldwell ME, Allard D, Frese KK, Denicola G, Feig C, Combs C, Winter SP, Ireland-Zecchini H, et al. Inhibition of Hedgehog signaling enhances delivery of chemotherapy in a mouse model of pancreatic cancer. Science. 2009; 324:1457-1461.

3. Ginsburg GS and McCarthy JJ. Personalized medicine: revolutionizing drug discovery and patient care. Trends Biotechnol. 2001; 19:491-496.

4. Nunn AD. Update: Molecular Imaging and Personalized Medicine: An Uncertain Future. Cancer Biother Radiopharm. 2007; 22:722-739.

5. Ward KM, Aletras AH and Balaban RS. A new class of contrast agents for MRI based on proton chemical exchange dependent saturation transfer (CEST). J Magn Reson. 2000; 143:79-87.

6. Liu G, Song X, Chan KW and McMahon MT. Nuts and bolts of chemical exchange saturation transfer MRI. NMR Biomed. 2013; 26:810-828.

7. van Zijl PC and Yadav NN. Chemical exchange saturation transfer (CEST): what is in a name and what isn't? Magn Reson Med. 2011; 65:927-948.

8. Walker-Samuel S, Ramasawmy R, Torrealdea F, Rega M, Rajkumar V, Johnson SP, Richardson S, Goncalves M, Parkes HG, Arstad E, Thomas DL, Pedley RB, Lythgoe $\mathrm{MF}$ and Golay X. In vivo imaging of glucose uptake and metabolism in tumors. Nat Med. 2013; 19:1067-1072.

9. Chan KW, McMahon MT, Kato Y, Liu G, Bulte JW, Bhujwalla ZM, Artemov D and van Zijl PC. Natural D-glucose as a biodegradable MRI contrast agent for detecting cancer. Magn Reson Med. 2012; 68:1764-1773.

10. Cai K, Haris M, Singh A, Kogan F, Greenberg JH, Hariharan H, Detre JA and Reddy R. Magnetic resonance imaging of glutamate. Nat Med. 2012; 18:302-306.

11. Goffeney N, Bulte JW, Duyn J, Bryant LH, Jr. and van Zijl PC. Sensitive NMR detection of cationic-polymer-based gene delivery systems using saturation transfer via proton exchange. J Am Chem Soc. 2001; 123:8628-8629.

12. Zhou J, Payen JF, Wilson DA, Traystman RJ and van Zijl PC. Using the amide proton signals of intracellular proteins and peptides to detect $\mathrm{pH}$ effects in MRI. Nat Med. 2003; 9:1085-1090.

13. Liu G, Liang Y, Bar-Shir A, Chan KW, Galpoththawela CS, Bernard SM, Tse T, Yadav NN, Walczak P, McMahon MT, Bulte JW, van Zijl PC and Gilad AA. Monitoring enzyme activity using a diamagnetic chemical exchange saturation transfer magnetic resonance imaging contrast agent. J Am Chem Soc. 2011; 133:16326-16329.

14. Liu G, Bettegowda C, Qiao Y, Staedtke V, Chan KW, Bai R, Li Y, Riggins GJ, Kinzler KW, Bulte JW, McMahon MT, Gilad AA, Vogelstein B, Zhou S and van Zijl PC. Noninvasive imaging of infection after treatment with tumor-homing bacteria using Chemical Exchange 
Saturation Transfer (CEST) MRI. Magn Reson Med. 2013; 70:1690-1698.

15. Friedman JI, McMahon MT, Stivers JT and Van Zijl PC. Indirect detection of labile solute proton spectra via the water signal using frequency-labeled exchange (FLEX) transfer. J Am Chem Soc. 2010; 132:1813-1815.

16. Liu G, Ali MM, Yoo B, Griswold MA, Tkach JA and Pagel MD. PARACEST MRI with improved temporal resolution. Magn Reson Med. 2009; 61:399-408.

17. Laprie A, Catalaa I, Cassol E, McKnight TR, Berchery D, Marre D, Bachaud JM, Berry I and Moyal EC. Proton magnetic resonance spectroscopic imaging in newly diagnosed glioblastoma: predictive value for the site of postradiotherapy relapse in a prospective longitudinal study. Int J Radiat Oncol Biol Phys. 2008; 70:773-781.

18. Verdun FR, Denys A, Valley JF, Schnyder P and Meuli RA. Detection of low-contrast objects: experimental comparison of single- and multi-detector row CT with a phantom. Radiology. 2002; 223:426-431.

19. Aime S, Castelli DD, Crich SG, Gianolio E and Terreno E. Pushing the sensitivity envelope of lanthanide-based magnetic resonance imaging (MRI) contrast agents for molecular imaging applications. Acc Chem Res. 2009; 42:822-831.

20. Ruiz-Cabello J, Walczak P, Kedziorek DA, Chacko VP, Schmieder AH, Wickline SA, Lanza GM and Bulte JW. In vivo "hot spot" MR imaging of neural stem cells using fluorinated nanoparticles. Magn Reson Med. 2008; 60:1506-1511.

21. Federico C, Morittu VM, Britti D, Trapasso E and Cosco D. Gemcitabine-loaded liposomes: rationale, potentialities and future perspectives. Int J Nanomedicine. 2012; 7:54235436.

22. Grazia Calvagno M, Celia C, Paolino D, Cosco D, Iannone M, Castelli F, Doldo P and Fresta M. Effects of lipid composition and preparation conditions on physicalchemical properties, technological parameters and in vitro biological activity of gemcitabine-loaded liposomes. Curr Drug Deliv. 2007; 4:89-101.

23. Liu G, Moake M, Har-el YE, Long CM, Chan KW, Cardona A, Jamil M, Walczak P, Gilad AA, Sgouros G, van Zijl PC, Bulte JW and McMahon MT. In vivo multicolor molecular MR imaging using diamagnetic chemical exchange saturation transfer liposomes. Magn Reson Med. 2012; 67:1106-1113.

24. Chan KW, Liu G, Song X, Kim H, Yu T, Arifin DR, Gilad AA, Hanes J, Walczak P, van Zijl PC, Bulte JW and McMahon MT. MRI-detectable $\mathrm{pH}$ nanosensors incorporated into hydrogels for in vivo sensing of transplanted-cell viability. Nat Mater. 2013; 12:268-275.

25. Chan KW, Yu T, Qiao Y, Liu Q, Yang M, Patel H, Liu G, Kinzler KW, Vogelstein B, Bulte JW, van Zijl PC, Hanes J, Zhou S and McMahon MT. A diaCEST MRI approach for monitoring liposomal accumulation in tumors. J Control
Release. 2014; 180:51-59.

26. Ma X, Song Y, Zhang K, Shang L, Gao Y, Zhang W, Xue X, Jia H, Geng J, Zhou W, Dang Y, Li E, Ti X, Fan F, Zhang $\mathrm{Y}$ and Li M. Recombinant mutated human TNF in combination with chemotherapy for stage IIIB/IV non-small cell lung cancer: a randomized, phase III study. Sci Rep. 2015; 4:9918.

27. Davis ME, Chen ZG and Shin DM. Nanoparticle therapeutics: an emerging treatment modality for cancer. Nat Rev Drug Discov. 2008; 7:771-782.

28. Sumer B and Gao J. Theranostic nanomedicine for cancer. Nanomedicine (Lond). 2008; 3:137-140.

29. Liu Z, Dimitrov IE, Lenkinski RE, Hajibeigi A and Vinogradov E. UCEPR: Ultrafast localized CESTspectroscopy with PRESS in phantoms and in vivo. Magn Reson Med. 2015.

30. Liu G, Chan KW, Song X, Zhang J, Gilad AA, Bulte JW, van Zijl PC and McMahon MT. NOrmalized MAgnetization Ratio (NOMAR) filtering for creation of tissue selective contrast maps. Magn Reson Med. 2013; 69:516-523.

31. Chen LQ, Howison CM, Jeffery JJ, Robey IF, Kuo PH and Pagel MD. Evaluations of extracellular $\mathrm{pH}$ within in vivo tumors using acidoCEST MRI. Magn Reson Med. 2014; 72:1408-1417.

32. Liu G, Gilad AA, Bulte JW, van Zijl PC and McMahon MT. High-throughput screening of chemical exchange saturation transfer MR contrast agents. Contrast Media Mol Imaging. 2010; 5:162-170.

33. Kim M, Gillen J, Landman BA, Zhou J and van Zijl PC. Water saturation shift referencing (WASSR) for chemical exchange saturation transfer (CEST) experiments. Magn Reson Med. 2009; 61:1441-1450. 